\title{
Direct measurements of structural forces and twist transitions in cholesteric liquid crystal films with the Surface Force Apparatus
}

Received 00th January 20xx, Accepted 00th January 20xx

DOI: $10.1039 / x 0 x x 00000 x$

\begin{abstract}
Weichao Zheng, ${ }^{a}$ Carla Perez-Martinez, ${ }^{\text {b }}$ Gia Petriashvili, ${ }^{c}$ Susan Perkin ${ }^{\text {b }}$ and Bruno Zappone $* d$
\end{abstract}
\section{Introduction}

Cholesteric liquid crystals (CLC) are one-dimensional periodic anisotropic materials with a photonic band-gap, polarizationselective optical reflectivity and high responsiveness to external stimuli. ${ }^{1,} 2$ These properties are widely used in applications such as thermal sensors, ${ }^{3-5}$ active optical filters and mirrors, 6-9 smart windows and displays, ${ }^{10}, 11$ mirror-less lasers ${ }^{12}$ and active surface coatings ${ }^{13-15}$. Typical CLCs comprise elongated chiral molecules that tend to align parallel to each other along a common director $\mathbf{n}$ with a spontaneous twist. Namely, $\mathbf{n}$ tends to rotate about a helical axis $z \perp \mathbf{n}$ with a uniform rotation rate $\partial \varphi / \partial z=\pi / p$ when the CLC is not deformed, where the half-pitch length $p$ is the natural period of the chiral structure (Fig. 1a). CLCs are optically anisotropic with the band-gap extending from wavelength $\lambda_{\mathrm{e}}=$ $\left(\pi n_{\mathrm{e}}\right)(\partial \varphi / \partial z)^{-1}$ to $\lambda_{\mathrm{o}}=\left(\pi n_{\mathrm{o}}\right)(\partial \varphi / \partial z)^{-1}$, where $n_{\mathrm{e}}$ and $n_{\mathrm{o}}$ are respectively the extraordinary and ordinary refractive indices. ${ }^{2}$ Most CLC applications rely on the stabilization, detection or active control of the rotation rate $\partial \varphi / \partial z$ as a function of the temperature, ${ }^{16-18}$ concentration of chiral dopants, film thickness and applied mechanical stress, ${ }^{19-21}$ surface anchoring potential,22, 23 electro-magnetic fields ${ }^{6,} 10,24$ and light irradiation ${ }^{25,26}$.

Here we introduce the Surface Force Apparatus - SFA 27 (also known as Surface Force Balance - SFB ${ }^{28}$ ) (Fig. 1b) as a powerful tool to study the structural and mechanical response of CLCs and other liquid crystal films to nanoscale confinement. ${ }^{29-46}$ We have recently demonstrated that the film

a. Università della Calabria, Dipartimento di Fisica, Rende (CS), Italy.

b. University of Oxford, Department of Chemistry, Physical and Theoretical Chemistry Laboratory, Oxford, United Kingdom.

c. Georgian Technical University, Institute of Cybernetics, Tbilisi, Georgia.

d. Consiglio Nazionale delle Ricerche, Istituto di Nanotecnologia (CNR-Nanotec), Rende (CS), c/o Dipartimento di Fisica, Università della Calabria, Via P. Bucci, 33/B, 87036 Rende (CS), Italy. thickness $D$, as well as the orientation and distortions of $\mathbf{n}$ can be determined by carefully analyzing the multiple-beam optical interference (MBI) data that are commonly used to determine $D$ in isotropic materials. ${ }^{46}$ Most importantly, the SFA allows to actively control the film thickness and induce structural transition in the CLC while simultaneously measuring structural forces acting on the confining surfaces. Compared to Atomic Force Microscope (AFM) measurements, ${ }^{47}$ the SFA has the additional advantage that the film thickness, surface curvature and anchoring conditions can be precisely determined, and experiments can be conducted at very slow surface velocity, letting the CLC equilibrate in response to thickness variations.

In our study, the CLC was confined under strong planar anchoring conditions between crossed-cylindrical surfaces with radius $R \gg p$ (Fig. $1 \mathrm{~b}$ ). The CLC film thickness can be approximated as $h(r)=D+r^{2} / 2 R$ where $r$ is the lateral distance (radius) from the contact point ( $r=0$ in Fig. 1b) and $D \ll R$ is the surface separation distance.
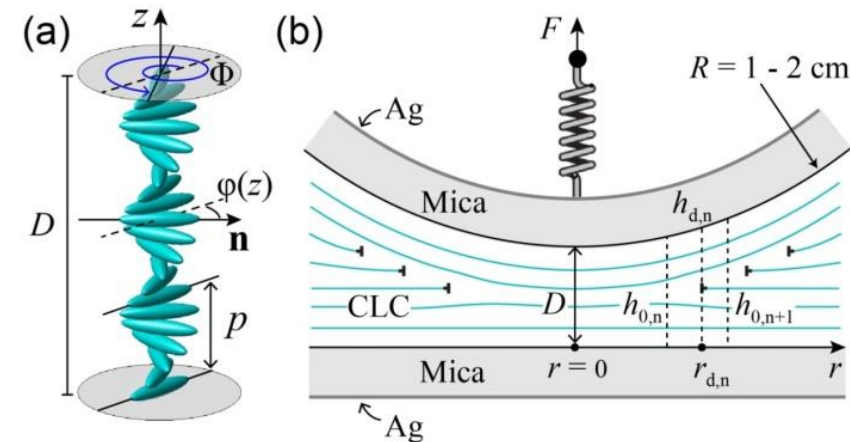

FIG. 1. (a) CLC structure in a film of thickness $D$ with planar anchoring conditions. $\mathbf{n}$ is the director, $\varphi$ the azimuthal angle, $p$ the half-pitch and $\Phi$ the total twist angle. (b) Side view of the crossed cylindrical mica surfaces with radius $R=(1-2) \mathrm{cm}$ at distance $D$ apart used in SFA experiments. $F$ is the normal force. T-symbols indicate dislocation loops. 
For $r / R<<1, \mathbf{n}$ is approximately parallel to the surfaces, and the helical axis is uniform and parallel to the surface normal $z$. When the planar anchoring directions on the two surfaces have a mismatch angle $\alpha$, the director $\mathbf{n}$ must rotate by a total twist angle $\Phi_{n}=n \pi+\alpha$ across the film thickness $h$ to satisfy both anchoring conditions. At equilibrium, the integer $n$ minimizes the free elastic energy, which is proportional to the difference $\left(\Phi_{\mathrm{n}} / h-\pi / p\right)^{2}$ between the actual rotation rate $\Phi_{\mathrm{n}} / h$ and the natural value. ${ }^{1} \mathrm{~A}$ twist transition between the angles $\Phi_{\mathrm{n}}$ and $\Phi_{\mathrm{n}} \pm \pi$ (i.e. between $n$ and $n \pm 1$ ) occurs when they produce the same elastic energy, corresponding to a critical thickness $h_{\mathrm{d}}(n) \approx p(n \pm 1 / 2)$ and radius $r_{\mathrm{d}}(n)^{2}=2 R\left[h_{\mathrm{d}}(n)-D\right]$ (Fig. 1b). ${ }^{48}$

In a film with non-uniform thickness, a twist transition marks the boundary between two CLC domains with different values of $n$ and occurs via the nucleation of a dislocation defect. An array of parallel dislocation lines is formed in a wedge-shaped film, showing the characteristic CanoGrandjean texture between crossed polarizer (Fig. 2a). ${ }^{1,49,50 ~ I n ~}$ the crossed-cylinder geometry of the SFA, which is equivalent to a sphere-plane geometry around the contact point, ${ }^{51}$ dislocations have an approximately circular shape with radius $r_{\mathrm{d}}$ centered at the contact point (Fig. 2b). Cano-Grandjean textures have been studied in great details for fixed confinement conditions. ${ }^{22,23,52-58}$ In the present work, we focused on the response of the CLC to changes of confinement thickness, and measured both the twist angle $\Phi$ and normal force as the surface separation distance $D$ was varied.
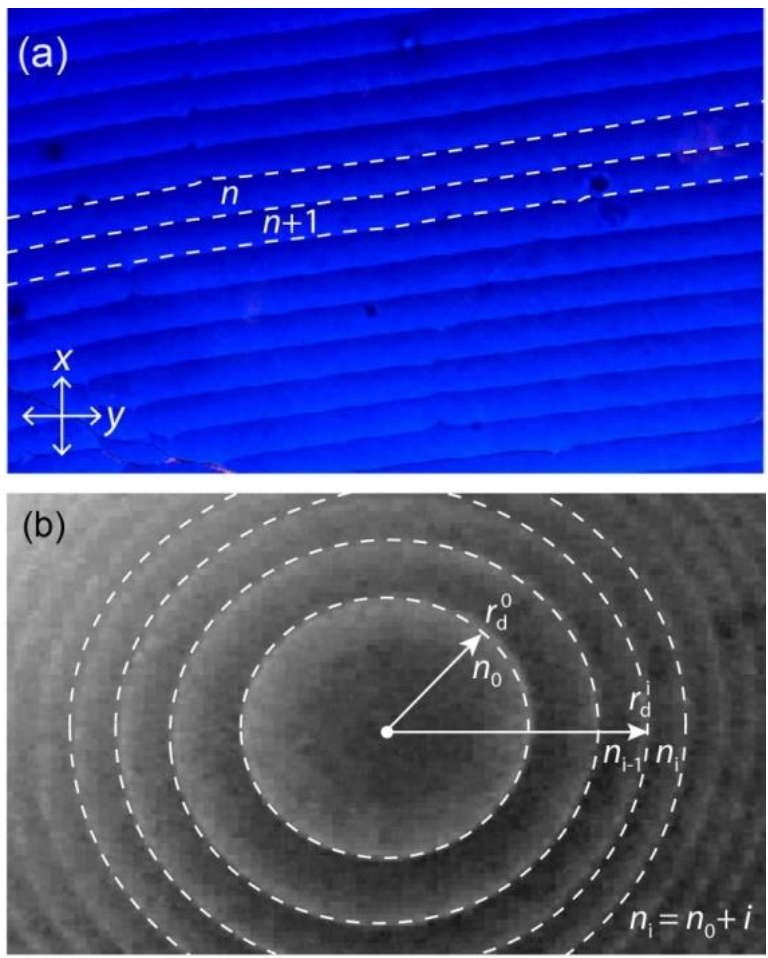

FIG. 2. Arrays of dislocation lines formed in a CLC confined under planar anchoring conditions between two mica surfaces with (a) wedge and (b) crossed cylinder confinement geometry. $n$ is the number of $\pi$ turns of the CLC director in the region comprised between two dislocations, indicated with dashed lines.
The dislocation loops shrank and eventually disappeared at the contact position as $D$ was increased. The disappearing of a loop corresponded to a twist transition from $n$ to $n+1$, and was observed at critical thicknesses $h_{\mathrm{d}}(n)$ periodically spaced by a half-pitch $p$. Twist transitions produced an oscillatory structural force that could be explained with a simple model of elastic deformation. Interestingly, the strength of the planar anchoring on mica was large compare to the values reported for common alignment surfaces such as oblique evaporated oxide layers and rubbed polymers ${ }^{52}$.

Our study was motivated by the idea that the band-gap of CLCs used in mirror-less lasers can be tuned via mechanical confinement of the CLC film. ${ }^{19,} 20$ Moreover, SFA can be used as a general platform to study confinement-induced and dynamic phenomena in CLC, e.g. helix inversion ${ }^{53-56}$, defect nucleation/annihilation and defect-mediated colloidal interactions, ${ }^{57}$ and permeative flow $48,58-61$. We anticipate that the behavior of the CLC observed during surface approach was very different from the one observed during separation. Notably, metastable states were observed due to the large energy required to nucleate new dislocations, which will be reported in a separate publication.

\section{Materials and methods}

\subsection{Surface Force Apparatus (SFA)}

In the SFA technique, the material under study is confined between two cylindrical glass lenses with radius $R=(1-2) \mathrm{cm}$ crossed at $90^{\circ}$ (Fig. 1b). The lenses are covered with cleaved sheets of muscovite mica with equal thickness $T$ of the order of a few $\mu \mathrm{m}$. The sheets are hard yet flexible, molecularly smooth, optically transparent and birefringent under normal incidence. ${ }^{62}$

We used a SFA Mark III (by Surforce LLC, USA) and a homemade SFB, described in details in refs 27,28 . The mica sheets were coated on one face with a semi-reflective 40-55 $\mathrm{nm}$ silver layer and glued with the coated side down on the glass lenses using a hard thermosetting glue (EPON 10007 by Shell). The surfaces were illuminated in the contact region using white light from a halogen lamp with normal incidence. Constructive interference of resonant light waves undergoing multiple reflections between the metal mirrors produced a spectrum of selectively transmitted wavelengths $\lambda$ that was used to determine the mica thickness $T$, mica-mica separation distance $D$ as well as the angles specifying the alignment of birefringent mica sheets and CLC director orientation. ${ }^{46}$

One of the surfaces was attached to the free end of a double spring cantilever with known elastic constant in the range $K=(0.1-1.0) \mathrm{kN} / \mathrm{m}$. The opposite (fixed) end of the cantilever was displaced vertically at a fixed speed $u_{0}$ of a few $\mathrm{nm} / \mathrm{s}$ using a motorized precision actuator. The distance $D$ was increased from contact $(D=0)$ to a few $\mu \mathrm{m}$. The normal force between the surfaces was calculated as $F(D)=K\left\{\left(D-D_{0}\right)-u_{0}(t\right.$ - $\left.\left.t_{0}\right)\right\}$ where $D_{0}$ was a large separation distance, reached at 
time $t=t_{0}$, beyond which the force was constantly zero. This formula expresses the balance between the normal force $F$ generated by the CLC and the elastic force of the cantilever. Since $F$ generally increases non-monotonically with $D$, a mechanical instability may occur at minima where the force is $F_{a}$ and $d F / d D>K$, in which case the surfaces jump outward over a distance $F_{\mathrm{a}} / K$ towards the next stable region. ${ }^{51}$

\subsection{Cholesteric liquid crystal mixture (CLC)}

A right-handed CLC was obtained by mixing $62.4 \%$ wt. nematic liquid crystal (NLC) QYPDLC-036 and 37.6\% wt. chiral dopant R2011 (both from Qingdao QY Liquid Crystal Co., Ltd. Chengyang Qingdao China). The NLC was similar to BL036 from Merck and contained mainly alkyl-cyano-biphenyls. The CLC mixture was designed to have large birefringence and low temperature sensitivity of the chiral pitch. The half-pitch $p$ $\approx 122 \mathrm{~nm}$ was determined using a spectrophotometer (Avaspec 2048-2 by Avantes) by measuring the edge wavelengths $\lambda_{\circ}$ and $\lambda_{\mathrm{e}}$ of the photonic band-gap.

\subsection{Surface anchoring}

Surface anchoring was studied in closed cells between crossed polarizers. When the CLC mixture was inserted in a wedge cell made with two thick plates of freshly cleaved mica, a CanoGrandjean texture was observed, with stripes running perpendicular to the film thickness gradient (Fig. 2a). This texture is typical of a CLC having the chiral helix normal to the boundaries, i.e. with planar alignment of the director $\mathbf{n}$ at the surfaces. In the absence of chiral dopant, the nematic component (NLC) appeared untextured between two mica plates and showed a large birefringence consistent with a planar alignment (Fig. 3).
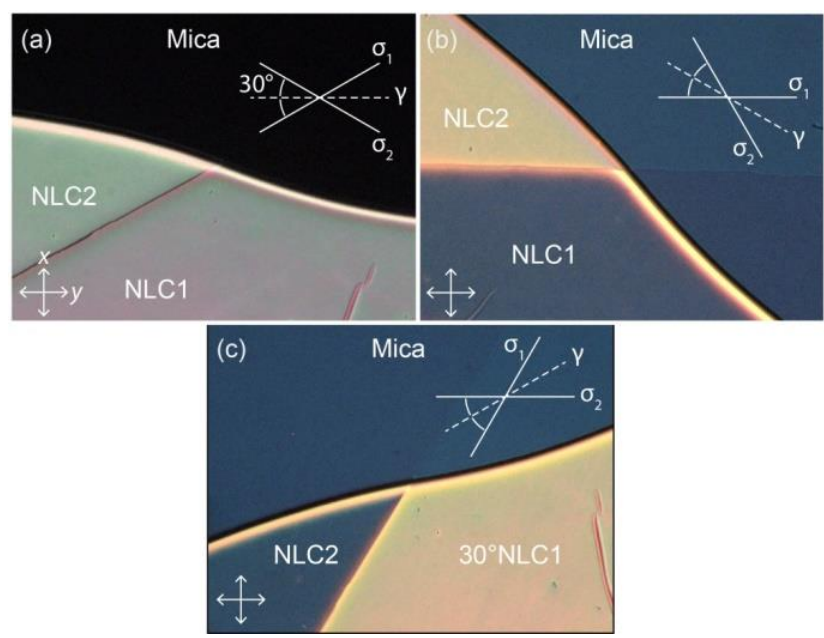

FIG. 3. (a) Edge of a nematic NLC drop confined between a thick mica plate and an OTS treated glass slide. The polarizer and analyzer were parallel to $x$ and $y$, respectively, and the mica index axis $\gamma$ was parallel to $y$. (b-c) The two domains NLC1 and NLC2 showed the same color as the mica plate when the sample was rotated by $+30^{\circ}$ or $-30^{\circ}$, respectively, corresponding to the angle between $\gamma$ and the planar anchoring directions $\sigma$ on different cleavage planes on mica.
To determine the angle between the in-plane anchoring direction and the mica index axis $\gamma$, droplets of NLC were confined under hybrid anchoring conditions between a thick mica plate and a glass slide coated with a monolayer of octadecyl-trichloro-silane (OTS) that induced normal (homeotropic) anchoring (Fig. 3). ${ }^{63}$

In regions with uniform film thickness, the NLC showed two types of uniform domains with different colors, corresponding to two different planar anchoring directions $\sigma$ depending on the mica cleavage plane. The domains had sharp boundaries corresponding to crystallographic steps on the mica surface.

To determine $\sigma$, first the direction of the $\gamma$ mica index axis was identified using a Berek-type compensator (from Leica, Germany). Namely, empty regions of the cell surrounding the NLC drop appeared dark when $\gamma$ was set at $\pm 45^{\circ}$ from the analyzer and the index axis of the compensator was inserted at $90^{\circ}$ with $\gamma$, between the sample and analyzer. Without compensator, mica in the empty regions appeared dark when $\gamma$ was parallel to the analyzer, while regions filled with NLC showed the same bright color (Fig. 3a). When $\gamma$ was rotated by $+30^{\circ}$ from the analyzer, one type of NLC domain showed the same color the empty regions while the other domain type showed a different color (Fig. 3b). The domain colors were exchanged when $\gamma$ was rotated by $-30^{\circ}$ from the analyzer (Fig. $3 c)$.

The color observed between crossed polarizer depends on the intensity $\left|E_{y}\right|^{2}$ of electric field $\left(E_{x}, E_{y}\right)$ transmitted through the analyzer, parallel to the $y$ direction. If a region filled with NLC transmits the same intensity $\left|E_{y}\right|^{2}$ as an empty region, the $2 \times 2$ Jones transmission matrix of the NLC must be diagonal in the $x y z$ reference frame, i.e. the director $\mathbf{n}$ to be either parallel or perpendicular to the $y z$ plane. Therefore, the anchoring direction $\sigma$ made an angle $\pm 30^{\circ}$ or $\pm 60^{\circ}$ with $\gamma$ in a NLC domain that appeared of the same color as empty regions upon rotating $\gamma$ by $\pm 30^{\circ}$. The actual angle between $\sigma$ and $\gamma$ was determined using the compensator. Namely, when $\gamma$ was parallel to the analyzer and the compensator axis was at right angles with one of the directions at $\pm 30^{\circ}$ with $\gamma$, one type of NLC domain could be extinguished. This shows that the angle between $\sigma$ and $\gamma$ was $\pm 30^{\circ}$, as previously observed for other thermotropic LCs based on alkyl-cyano-biphenyls. ${ }^{52,}$, 22, 64-66

\subsection{Analysis of multiple-beam interferometry (MBI)}

The analysis of the transmitted spectra due to $\mathrm{MBI}$ between the silver mirrors provides important information not only on the thickness $D$ of the CLC film at the contact position, but also on the alignment of the director $\mathbf{n}$ and total twist angle $\Phi$ (Fig. 1a). We used an imaging spectrograph coupled to a CCD camera to measure the MBI transmitted spectra at the contact position. Figure 4a shows a typical spectrograph image where the transmitted intensity is resolved as a function of the wavelength $\lambda$ along the direction $r$ parallel to one of the SFA cylinder axis. 


\section{ARTICLE}
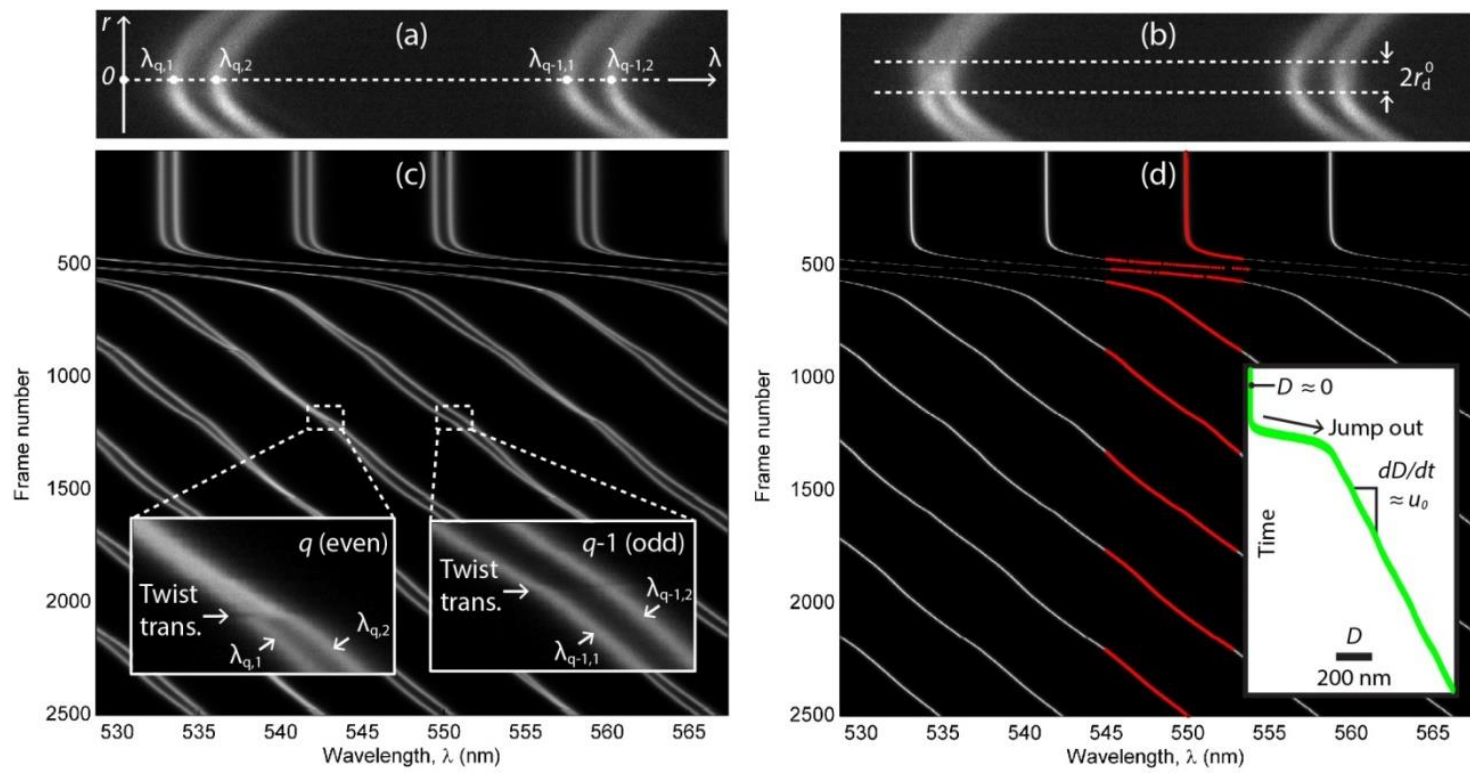

FIG. 4. (a) CCD camera frame showing the transmitted MBI intensity as a function of the wavelength $\lambda$ and lateral distance $r$ from the contact position ( $r=0$ ). FECOs appear as curved doublet lines. (b) Observation of the inner dislocation loop with (minimum) radius $r_{d}{ }^{0}$ (dashed lines). (c) MBI spectrogram of the intensity transmitted at contact position during surface retraction, resolved as a function of $\lambda$ and camera frame, proportional to the elapsed time. Twist transitions appear as sudden changes of the doublet splitting depending on the chromatic order $q$. (d) MBI spectrogram of the equivalent isotropic layer calculated from the the surface separation distance $D$ (Inset) obtained from the measured average wavelengths $\lambda_{\mathrm{q}}$ (red dots). $u_{0}$ is the constant speed of cantilever displacement.

The image shows a sequence of curved fringes of equal chromatic order (FECO), each corresponding to the resonance wavelengths $\lambda_{q}(r)$ with the chromatic order $q$. FECOs appeared curved because $\lambda_{q}$ increases as the surface separation distance $h(r)$ increases, and the latter increases as a function of $r$ in the crossed-cylinder geometry. Moreover, in the presence of birefringent materials such as mica and liquid crystals, the resonance condition for a given order $q$ is satisfied by two distinct polarization eigenstates, respectively at wavelengths $\lambda_{q, 1}(r)$ and $\lambda_{q, 2}(r)$, forming a doublet. ${ }^{67}$

A dislocation loop with radius $r_{\mathrm{d}}$ (Figs $1 \mathrm{~b}$ and $2 \mathrm{~b}$ ) can be directly observed in the spectrograph images as a sudden variation of the FECO splitting $\lambda_{\mathrm{q}, 1}-\lambda_{\mathrm{q}, 2}$ at points with $r= \pm r_{\mathrm{d}}$, i.e. above and below the contact position ( $r=0$, Fig. 4b). A spectrogram (Fig. 4c) is a sequence of $\mathrm{MBI}$ spectra transmitted at the contact position at different times (i.e. obtained from consecutive CCD camera frames during a continuous acquisition). In a spectrogram, the transmitted intensity is shown as a function of wavelength $\lambda$ and time, and FECOs appear as lines. Discontinuous twist transitions at the contact position, corresponding to the annihilation of a defect loop during surface retraction, appear as interruptions of the FECO lines.

A common problem in the SFA studies of optically anisotropic materials such as liquid crystals and mica is that the slow index axes $\gamma$ of the two mica surfaces generally form an oblique angle $\Psi$, and the direction of planar anchoring on the surface is oblique to $\gamma$. Standard MBI analysis derived for isotropic material68, 69 and used in most SFA experiments cannot capture the variety and complexity of the possible alignments and director distortions, and may lead to an incorrect determination of $D$. A new method has been recently developed to analyze $\mathrm{MBI}$ spectra of twisted NLC and CLC based on $4 \times 4$ transfer matrix multiplication. 46 It has been shown that the average value $\lambda_{q}=\left(\lambda_{q, 1}+\lambda_{q, 2}\right) / 2$ generated by a CLC with planar alignment closely approximates the wavelength $\lambda_{q}$ iso that would be generated if the mica sheets and CLC film were optically isotropic, with an average refractive index $\left(n_{\mathrm{o}}+n_{\mathrm{e}}\right) / 2 .{ }^{46}$ The distance $D$ can therefore be calculated from the measured averages $\lambda_{q}$ using the formulae derived for isotropic fluids ${ }^{68,} 69$ and refractive index values taken from the literature. Figure $4 d$ shows that, indeed, simulated $\mathrm{MBI}$ spectra of the equivalent isotropic layers had peak intensities at wavelength $\lambda_{q}{ }^{\text {iso }}$ very close to the values $\lambda_{q}$ measured for the CLC.

When the mica surfaces are in direct contact $(D=0)$, the average wavelength $\lambda_{\mathrm{q}}$ depends only on the mica thickness $T$, while the splitting $\Delta \lambda_{q}$ depends on $T$ and the angle $\Psi$ between the $\gamma$ index axes according to formula $\left|\Delta \lambda_{q}(\Psi)\right|=\left|\Delta \lambda_{q}(0)\right| \cos ^{2} \Psi .27,67$ The value of $T$ and $|\Psi|$ could therefore be determined from measured values of $\lambda_{q}$ and $\Delta \lambda_{q}$, using the known refractive indices of mica ${ }^{68}$ and silver ${ }^{70}$. Note that one value of $|\Psi|$ generally corresponds to multiple possible values of the anchoring mismatch $\alpha$ angle because the anchoring directions at the two surfaces can make an angle $\pm 30^{\circ}$ with the $\gamma$ axes (Fig. $5 \mathrm{a}$ and 7 ). For $D>0$, the doublet 
splitting $\Delta \lambda_{q}=\lambda_{q, 1}-\lambda_{q, 2}$ is sensitive to the alignment of the director $\mathbf{n}$ relative to the mica $\gamma$ axes and twist angle $\Phi .{ }^{46}$ This was used to perform a $\mathrm{MBI}$ analysis of twist transitions obtained at the contact position during surface retraction (Fig. 4c).

\section{Theoretical background}

\subsection{Twist transitions between plane parallel surfaces}

We consider a right-handed CLC film confined between two planar parallel mica plates with surface normal $z$, located at $z=$ 0 and $z=h$. The planar anchoring directions had a mismatch angle $\alpha \in(-\pi / 2, \pi / 2]$ between the bottom and top mica surface (Fig. 5a, Inset). The director $\mathbf{n}$ is assumed to rotate about $z$ while remaining parallel to the surfaces. The anchoring conditions are satisfied for twist angles $\Phi_{0}(n)=\alpha+n \pi$, where the natural number $n$ indicates how many times $\mathbf{n}$ rotates by $\pi$ (half turn) across the film thickness. Note that angles are taken with sign according to the usual right-hand rule relative to $z$, and the angles $\Phi$ and $-\Phi$ describe distinct states of alignment. When the anchoring strength is finite, the director $\mathbf{n}$ can deviate from the planar anchoring directions at the surfaces. We consider the symmetric case where both surfaces have the same azimuthal anchoring strength $W$. Other cases have been studied in detail in the literature.23, 48, 61, 71 Because of the symmetry, the deviation angles at the bottom and top surface have the same amplitude $\delta$ but opposite sign (Fig. 5a, Inset). The free elastic energy per unit area of the film can be written as: 48

$\mathrm{G}=\left(K_{22} / 2\right)(\Phi / h-\pi / p)^{2} h+W \sin ^{2} \delta$

where $K_{22}$ is the twist elastic constant, $\Phi$ is the total twist angle and $\pi / p$ is the rate of spontaneous (azimuthal) rotation of the CLC. We have assumed the usual Rapini-Papoular anchoring potential (second term in Eq. 1), that is valid in the limit of small $\delta$. This surface deviation can be expressed as:

$\delta=\left[\Phi_{0}(n)-\Phi\right] / 2 \in(-\pi / 2, \pi / 2]$

When $W$ is finite, the anchoring energy is minimized when $\delta$ is minimum. This corresponds to choosing the value of $\Phi_{0}$ (i.e. of $n$ ) closest to $\Phi$ in Eq. 2. Therefore $n$ can be expressed as a function of $\Phi$ and the free energy $G$ can be considered a function of $h$ and $\Phi$. The equilibrium twist angle $\Phi(h)$ and minimum energy $G_{0}(h)$ of a CLC film with given thickness $h$ can be determined from the equilibrium condition $\partial G(h, \Phi) / \partial \Phi=0$ using simple numerical methods (Fig. 5). Note that $G$ is defined positive and has zeros at thicknesses such that $h_{0}(n) / p=$ $\Phi_{0}(n) / \pi$, i.e. $G_{0}\left(h_{0}(n)\right)=0$.

In a CLC film with given thickness $h$, a twist transition can be triggered by thermal fluctuations from angles $\Phi$ and $\delta$ to different angles $\Phi^{\prime}$ and $\delta^{\prime}$ if the two state of alignment have the same energy $G(h)$. Equation 1 shows that this can happen at a critical thickness $h_{\mathrm{c}}$ such that $\left(\Phi^{\prime} / \pi-h_{c} / p\right)^{2}=\left(\Phi / \pi-h_{c} / p\right)^{2}$ and $\sin ^{2}\left(\delta^{\prime}\right)=\sin ^{2}(\delta)$.
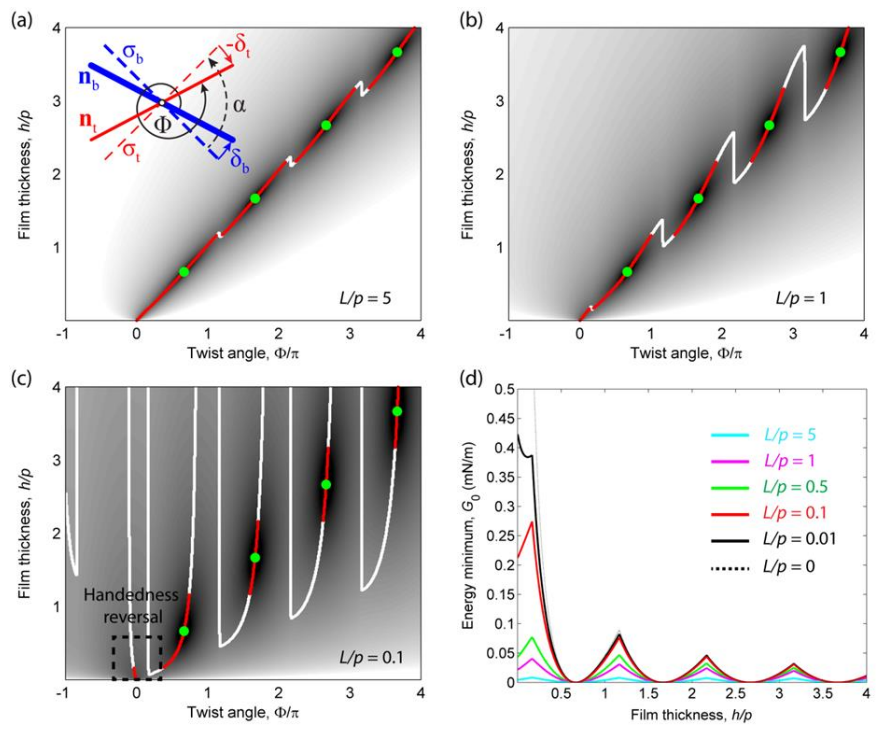

FIG. 5. (a-c) Logarithm of the normalized free energy $G / W$ calculated as a function of the normalized CLC film thickness $h / p$ and total twist $\Phi$ for a surface anchoring mismatch angle $\alpha=-\pi / 3$ and various extrapolation length $L$. Zeros, minima and extrema of $G$ are indicated with green dots, red lines and white lines, respectively. The inset in (a) shows the surface director $\mathbf{n}$ (solid lines) deviating by an angle $\pm \delta$ from the planar anchoring directions $\sigma$ (dashed lines) at the bottom and top mica surface (labeled ' $b$ ' and ' $t$ ' respectively). $\Phi$ is an example of total twist angle. The handedness is reversed in the dashed rectangle of (c). (d) Free energy minimum $G_{0}$ as a function of $h / p$ calculated for a twist elastic constant $K_{22}=10 \mathrm{pN}$ and increasing values of $L$ (indicated with different colored lines).

These conditions are satisfied when $\delta^{\prime}=-\delta$ and $\left(\Phi^{\prime}+\Phi\right) / 2=\alpha+$ $\left(n^{\prime}+n\right) \pi$, leading to the equation $h_{c} / p=\left(\Phi^{\prime}+\Phi\right) / 2 \pi$. Considering transitions between states with $n^{\prime}=n \pm 1$, we obtain $h_{\mathrm{c}}=p(\alpha / \pi+n \pm 1 / 2)=h_{0}(1 \pm 1 / 2)$. In other words, the critical thickness $h_{\mathrm{c}}$ is at the midpoint between two neighboring zero-energy thicknesses $h_{0}$. Twist transitions are generally discontinuous and the twist angle jumps by $\Phi^{\prime}-\Phi=$ $-2[(\Phi-\alpha+n \pi \pm \pi / 2]=-4 \delta \pm \pi$.

The study of Eq. 1 shows that the response of the CLC to variations of $h$ mainly depends on the anchoring extrapolation length $L=K_{22} / W$. For a rotating director field with constant rate $\Phi / h$, this can be thought as the depth within the substrate where the director orientation would virtually match the anchoring condition. ${ }^{1}$ For $h>L$, deviations from anchoring directions are negligible and the anchoring can be considered strong. It is worth noting that when the number of half turns reaches $n=0$ with $\alpha<0$, strong anchoring may lead to a severe frustration of the helical structure in a right-handed CLC. Indeed, at the transition from $n=1$ to $n=0$ the director rotation must reverse to a left-handed helical structure with total twist angle $\Phi=\alpha$ to satisfy both anchoring conditions (Fig. 5c, Inset). In the opposite case $h \ll L$, the anchoring is effectively weak. The director freely rotates at the surfaces and the anchoring energy increases, while the rotation rate $\Phi / h=\pi / p$ remains close to its natural value and the elastic energy close to zero. At the critical thickness $h_{c}$, the twist angle changes continuously while the surface deviation discontinuously jumps by $\pm \pi / 2$.

Figure $5 d$ shows the energy minimum $G_{0}(h)$ for various extrapolation lengths $L$. $G_{0}$ periodically oscillates with a 
decaying amplitude as $h$ increases. When $L$ decreases, the oscillation amplitude increases and the decay length decreases. Also, $G_{0}$ tends to a finite value for $h / p \rightarrow 0$ for finite values of $L / p$ and diverges in the limit $L / p \rightarrow 0$.

\subsection{Force generation between curved surfaces}

In a CLC film with small thickness and small thickness gradient, dislocations have Burgers vector of magnitude $p$ and are located at the midpoint between the boundaries (Fig. 1b). ${ }^{1,50}$ In the SFA geometry, the behavior of dislocation loops and structural force generation in response to thickness changes have been considered by Richetti et al for a smectic (lamellar) liquid crystal having the $1 \mathrm{~d}$-periodic modulation of molecular density normal to the surfaces (analogue to the CLC helical axis). ${ }^{32}$ The CLC film can be divided in a series of concentric cylindrical cells with axis $z$ normal to the surfaces and centered at the contact point (Figs $1 \mathrm{~b}$ and $2 \mathrm{~b}$ ). The central cell of order $i$ $=0$ has $n_{0}$ helical half-turns and extends from $r=0$ to the radius $r_{0}\left(n_{0}\right)$ where the surface separation distance is equal to the zero-energy thickness $h_{0}\left(n_{0}\right)$ derived from Eq. 1 . The outer cell of order $i>0$ goes from $r_{0}\left(n_{\mathrm{i}}-1\right)$ to $r_{0}\left(n_{\mathrm{i}}\right)$, where $n_{\mathrm{i}}=n_{0}+i$, and contains a circular dislocation loop with radius $r_{\mathrm{d}}\left(n_{\mathrm{i}}\right)$. The loop marks the distance from the contact point where the film thickness reaches the critical value $h_{\mathrm{d}}\left(n_{\mathrm{i}}\right)$ for the twist transition from $n_{\mathrm{i}}-1$ to $n_{\mathrm{i}}$

Assuming that the dislocations do not interact with each other, the energy in the CLC film can be written as $E=E_{0}+\Sigma\left\{E_{\mathrm{i}}+\right.$ $\left.2 \pi \mu r_{\mathrm{d}}\left(n_{\mathrm{i}}\right)\right\}$ with $i>0$, where $E_{0}$ and $E_{\mathrm{i}}$ are the elastic energies of defect-free regions, and $2 \pi \mu r_{d}\left(n_{i}\right)$ is due to the dislocation loop with line tension $\mu$. The elastic energy terms are given by:

$$
\left\{\begin{array}{l}
E_{0}=\int_{0}^{r_{0}^{0}} 2 \pi r d r G\left\{h(r), n_{0}\right\} \\
E_{i}=\int_{r_{0}^{i-1}}^{r_{d}^{i}} 2 \pi r d r G\left\{h(r), n_{i}-1\right\}+\int_{r_{d}^{i}}^{r_{0}^{i}} 2 \pi r d r G\left\{h(r), n_{i}\right\}
\end{array}\right.
$$

where $r_{\alpha}{ }^{i}=r_{\alpha}\left(n_{i}\right)$ and $G\left\{h(r), n_{i}\right\}$ is the free energy per unit area (Eq. 1) for $n_{i}$ half-turns confined within a (non-uniform) film thickness $h$. The radius of the dislocations is obtained by minimizing $E$ with respect to $r_{d}$, i.e. $\partial E / \partial r_{d}{ }^{i}=\partial E_{i} / \partial r_{d}{ }^{i}+2 \pi \mu=0$. From Eq. 3 , this leads to the equilibrium condition $r_{d}{ }^{i} \Delta G_{i}=\mu$, where $\Delta G_{\mathrm{i}}=G\left(h_{\mathrm{d}}^{\mathrm{i}}, n_{\mathrm{i}}\right)-G\left(h_{\mathrm{d}} \mathrm{i}, n_{\mathrm{i}}-1\right)$ is the elastic energy cost of having a twist transition from $n_{\mathrm{i}}-1$ to $n_{\mathrm{i}}$ at the critical thickness $h_{\mathrm{d}}$. Note that $\mu=0$ entails $\Delta G_{\mathrm{i}}=0$, i.e. the condition for twist transition between plane parallel surfaces. Therefore, for $\mu=0$ the critical thickness is $h_{d}{ }^{i}=h_{c}\left(n_{i}-1\right)=p\left(\alpha / \pi+n_{i}-1 / 2\right)$. For $\mu>0$, the additional energy cost due to line tension is partially compensated by decreasing the length of the dislocation, i.e. reducing the loop radius: $r_{\mathrm{d}} \leq r\left(h_{\mathrm{c}}\right)$. This also entails that the critical thickness is reduced, $h_{\mathrm{d}} \leq h_{\mathrm{c}}$. For strong anchoring, it is straightforward to show from Eq. 1 that $\Delta G=$ $\left(K_{22} \pi^{2} / p\right)\left(h_{c} / h_{d}-1\right)$, leading to the condition:

$h_{\mathrm{c}} / h_{\mathrm{d}}-1=s / r_{\mathrm{d}}$ where $s / p=\left(\mu / K_{22} \pi^{2}\right)$. This equation shows that the effect of finite line tension is negligible as long as $r_{\mathrm{d}} \gg s$ and becomes significant as $r_{\mathrm{d}}$ decreases to values of the order of $s$. Since the line tension of a CLC is in the range $\mu / K_{22}=(1-10),{ }^{1,}, 50$ corresponding to $s=(0.1-1) p=(10-100) \mathrm{nm}$, the effect of finite line tension become significant when $r_{\mathrm{d}}$ decreases to a few hundred $\mathrm{nm}$. Such small radius and therefore the effect of line tension cannot be directly resolved by optical microscopy in the SFA. For $r_{\mathrm{d}} \gg s$, the radius is given by $r_{\mathrm{d}}(n)^{2} / 2 R \approx h_{\mathrm{c}}(n)-$ $D$ and the distance between consecutive loops is $(2 R p)^{1 / 2}\left\{n^{1 / 2}\right.$ $\left.(n-1)^{1 / 2}\right\}$ where $(2 R p)^{1 / 2} \sim 70 \mu \mathrm{m}$ (assuming $\alpha=0$ ). Therefore the distance between dislocations remains much larger than $p$ for $n$ as large as a few hundred. A dislocation produces a strain field that decays along the $r$ direction (perpendicular to both the helix and dislocation) over a distance of the order of $p .^{1}$ Therefore the first few hundred dislocations can be considered independent from each other, consistent with the initial assumption.

Figure 6 shows the dislocation radius $r_{\mathrm{d}}$ as a function of the surface distance $D$, calculated from Eq. 4 in the case of large line tension $(s=p)$, strong anchoring $(L=0)$ and parallel anchoring conditions $(\alpha=0)$.

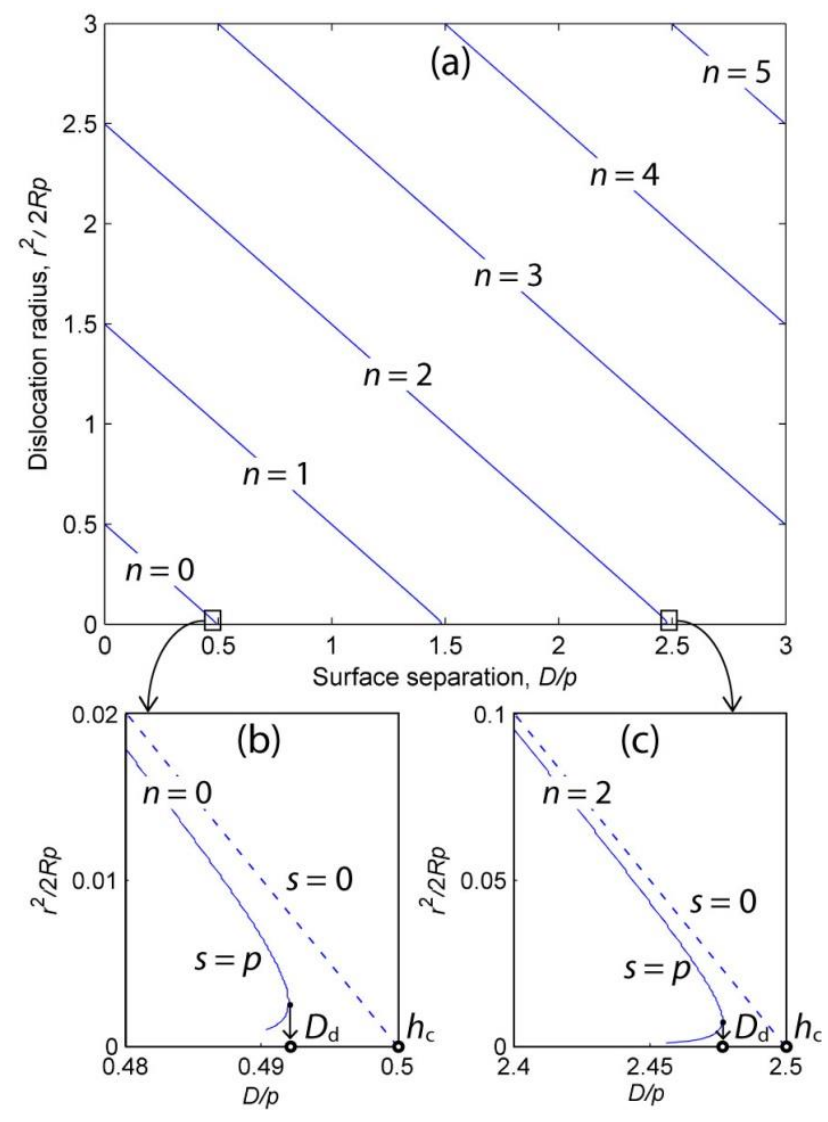

FIG. 6. (a) Equilibrium radius $r_{\mathrm{d}}$ of the dislocation loops as a function of the separation distance $D$ for a large line tension $(s=p)$ and strong parallel anchoring conditions $(L=0$, $\alpha=0$ ). The dislocation labeled $n$ marks the transition from $n$ to $n+1$ half-turns. (b-c) Enlarged view of loop disappearance during surface retraction. For a non-zero line tension, the loop radius discontinuously jumps to zero at $D=D_{\mathrm{d}}$ whereas for zero line tension it continuously decreases and reaches zero at $D=h_{\mathrm{c}}$. 
While for zero line tension the radius decreases during surface retraction and continuously reaches zero (i.e. the loop disappears) at the separation distance $D=h_{c}(n)$, in the presence of a non-zero line tension the radius discontinuously jumps to zero when the distance exceeds $D_{\mathrm{d}}(n)<h_{\mathrm{c}}(n)$. The difference $h_{\mathrm{c}}-D_{\mathrm{d}}$ decreases with $n$ and is of the order of $p / 10$ at most (i.e. for $n=0$ and large line tension). We therefore expect that the effects of a non-zero line tension to be rather weak and difficult to detect.

The normal force between the surfaces is $F=-d E / d D=-$ $\partial E / \partial D-\Sigma\left(\partial E / \partial r_{\mathrm{d}^{i}}\right)\left(\partial r_{\mathrm{d}^{i}} / \partial D\right)$. The last term is zero at equilibrium since the dislocation loops satisfy $\partial E / \partial r_{d}{ }^{i}=0$. Differentiation of the elastic energy in Eq. 3 gives:

$$
F / 2 \pi R=G_{0}(D)+\sum_{i} \Delta G_{i}=G_{0}(D)+\sum_{i} \mu / r_{d}^{i}
$$

where we have used the fact that $-\partial G / \partial D=-(R / r) \partial G / \partial r$ in the crossed-cylinder geometry. The first term is the force generated by the central cell and it has the oscillatory character shown in Fig. $5 \mathrm{~d}$. The second term is due to the line tension of dislocations with $i>0$. According to ref., the second term is small, non-oscillatory and slowly changing with $D$ over a distance of many pitch lengths. We therefore expect a nonzero line tension to add a small constant background to the force, which cannot be detected with the SFA.

Surface curvature in the crossed-cylinder geometry also affects the uniformity of anchoring conditions. If the mismatch angle between (co-planar) anchoring directions is $\alpha$ at the contact position ( $r=0$ ), they will generally be non co-planar and twisted at different angle at any other point. ${ }^{29,} 44$ However, the deviation angle from the common plane and variation of mismatch angle are of the order of $r / R$. In our experiments, $\mathrm{MBI}$ observations and elastic force measurements concern the central cell of dislocation array, contained within a radius $r_{d}\left(n_{0}\right) \leq(p R)^{1 / 2} \approx 30 \mu \mathrm{m}$. Therefore, curvature-related angles are of the order of $10^{-3} \mathrm{rad}$ and can be neglected.

\section{Experimental results}

\subsection{Twist transitions}

The surfaces were separated from contact by displacing the fixed end of the SFA cantilever at a constant speed $u_{0}$. They initially remained in contact with each other due to surface adhesion, at a surface separation distance $D=(1-4) \mathrm{nm}$ (Fig. $4 \mathrm{~d}$, Inset). When the adhesive minimum was reached and the force $F$ become such that $\partial F / \partial D>K$, the surfaces jumped outwards with a speed $u>u_{0}$ until they reached the next stable region. As the surfaces were further separated, they moved with a speed $u \approx u_{0}$ indicative of a very weak force. The detailed force vs. distance curve will be presented in the next section.
As $D$ was increased, observation of the FECO fringes (Fig. 4b) showed that the radius $r_{d}$ of the inner dislocation loop decreased until the loop eventually disappeared at a critical surface separation distance $D_{\mathrm{d}}$ where a twist transition from $n_{0}$ to $n_{0}+1$ occurred at the contact position. During this process, the loop appeared to continuously shrink down to zero radius and we did not detect any discontinuity due to finite line tension $\mu$ (Eq. 4 and Fig. $6(\mathrm{~b}, \mathrm{c})$,$) ). Figure 7$ shows the critical thickness $D_{\mathrm{d}}$ as a function the number $n_{0}$ of half turns at the contact position. $D_{\mathrm{d}}$ increased linearly with $n_{0}$ as expected for a negligible line tension: $D_{\mathrm{d}} / p \approx h_{\mathrm{c}} / p=\alpha / \pi+n_{0}+1 / 2$. The slope $p=$ $(122 \pm 1) \mathrm{nm}$ was very close to the half-pitch value determined from the edge wavelengths of the photonic band-gap.

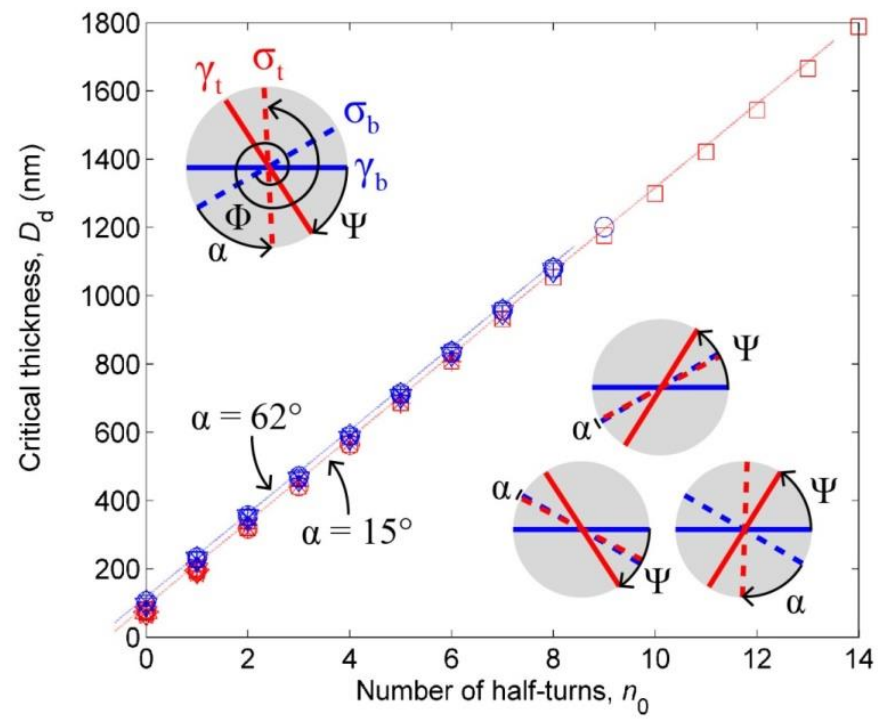

FIG. 7. Critical distance $D_{\mathrm{d}}$ for loop annihilation/twist transition as a function of the number $n_{0}$ of half turns at contact. Each color represents a different pair of mica surfaces, and each symbol a different measurement. The two lines are fits of the equation $D_{c} / p=\alpha / \pi+n_{0}+1 / 2$ (assuming zero line tension, $\mu=0$ ), with two different values of the mismatch angle: $\alpha=62.5^{\circ}$ and $\alpha=15.0^{\circ}$. The inset in the upper left corner shows the planar anchoring directions $\sigma$ for $\alpha=62.5^{\circ}$ that agrees with the measured angle $|\Psi|=57.5$ o. The twist angle $\Phi=\alpha+n \pi$ is shown for $n=3$. The inset in the lower right corner shows the other three possible values of $\alpha$ compatible with $|\Psi|$.

The linear dependence included the first three twist transitions $\left(n_{0}=0,1,2\right)$, detected in $\mathrm{MBI}$ spectrographs during surface jump-out (Fig. 4d, Inset). This indicates that the dynamics of twist transition was fast and unaffected by surface motion. Extrapolating $D_{\mathrm{d}}$ to $n_{0}=0$ we obtained the angle $\alpha=\pi\left(D_{\mathrm{d}} / p-\right.$ $1 / 2$ ) between the planar anchoring directions. This matched one of the four possible values compatible with the measured value $|\Psi|$ of the angle between mica $\gamma$ axes (Fig. 7, Inset).

\subsection{Anchoring extrapolation length}

In the limit of large extrapolation length, $L>p$, twist transitions should be continuous at surface distances such that $D \ll L$, particularly for small value of $n_{0}$ (Fig. 5(a-c)). In our experiments, all transitions were discontinuous, including the very first transition from $n_{0}=0$ to $n_{0}=1$, occurring at the critical distance $D_{\mathrm{d}}=(\alpha / \pi+1 / 2) p \leq p$. Therefore, the 
extrapolation length was $L \ll p$. At the transition, the FECO splitting $\lambda_{q, 1}-\lambda_{q, 2}$ suddenly varied by an amount that depended both on $n_{0}$ and the parity $q$ of the FECO chromatic order (Fig. $4 b$, Inset). In order to determine $L$, the experimental $M B I$ spectrograms were compared to the spectra obtained with the $4 \times 4$ matrix method ${ }^{46}$. We used the measured values of mica thickness $T$, angles $\Psi$ and $\alpha$, surface distance $D$ and half pitch $p$, whereas the total twist angle $\Phi(D)$ was calculated by minimizing $G(D, \Phi(D))$ in Eq. 1 . The simulations closely reproduce the $\mathrm{MBI}$ spectrographs, including the dependence of the FECO splitting on $n_{0}$ and $q$, for large extrapolation lengths $L / p \leq 0.1$ (Fig. 8)

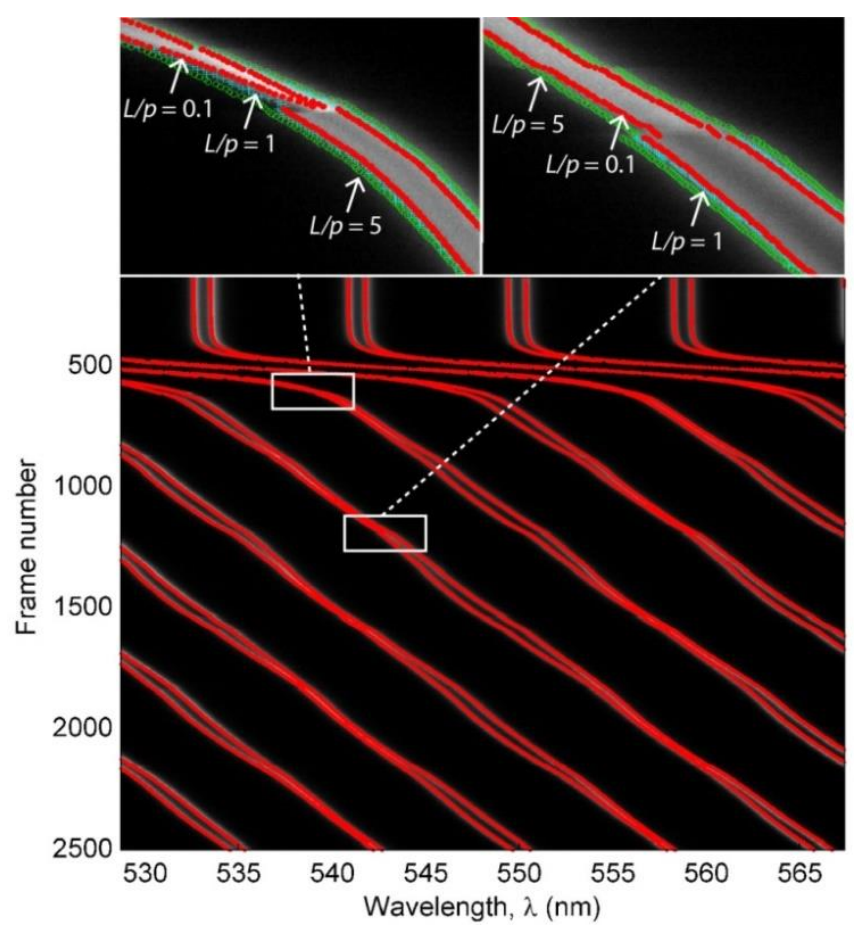

FIG. 8. Wavelength positions (red dots) of calculated MBI intensity peaks superposed to the experimental $\mathrm{MBI}$ spectrogram of Fig. 4c (grayscale image). The calculation parameters were: $\Psi=-57.4^{\circ}, \alpha=62.6^{\circ}$ (from Fig. 7), $p=122 \mathrm{~nm}, L / p=0.1$ and $K_{22}=10$ $\mathrm{pN}$. The two insets at the top of the image also show the wavelength positions near twist transitions calculated for $L / p=0.1$ (red dots), $L / p=1$ (cyan crosses) and $L / p=5$ (green circles).

Considering typical values of the twist elastic constant, $K_{22}=$ (3-10) $\mathrm{pN}, 72,73$ this corresponds to an azimuthal anchoring strength $W=K_{22} / L \geq 0.2 \mathrm{~mJ} / \mathrm{m}^{2}$. This value is at the higher end of the range reported for azimuthal planar anchoring strength $^{52}$ and comparable, e.g., with the anchoring of $5 C B$ on rubbed polymers measured with similar all-optical methods (without applied fields) ${ }^{74,75}$

\subsection{Structural forces}

The CLC deformation generated weak oscillatory forces surfaces that were close to the detection limit of the SFA setup (Fig. 9). The surface moved at a speed $u$ that varied periodically as $D$ was increased beyond the initial outward jump (Fig. 9a). Moreover, the normal force $F$ showed as series of periodic maxima at distances $D_{\mathrm{d}}$ where twist transitions where observed in $\mathrm{MBI}$ spectrograms, with normalized force amplitudes $F / R$ of the order of $(0.2-0.4) \mathrm{mN} / \mathrm{m}$, slightly above the sensitivity of the SFA setup (Fig. 9b). The amplitude of the force oscillations was consistent with the model of force generation of Eqs 1 and 5, with a strong azimuthal anchoring such that $L / p<0.1$. The force was adhesive when the surfaces were at a distance of a few $\mathrm{nm}$, with a rather strong adhesion force $F_{0}=(5-6) \mathrm{mN} / \mathrm{m}$ and long outward jump (Fig. 9c).

(a)

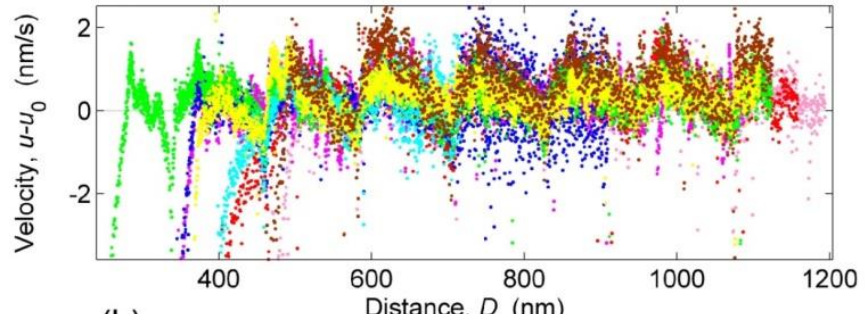

(b)

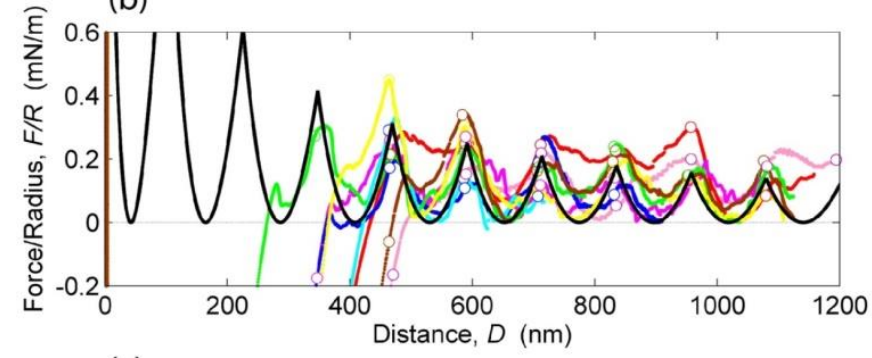

(c)

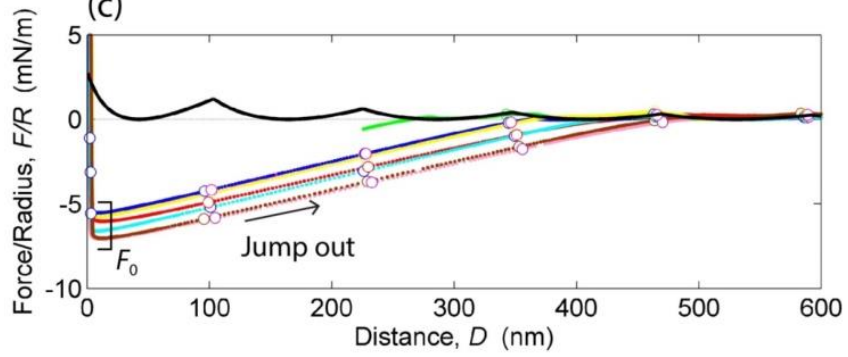

FIG. 9. (a) Change of surface velocity $u-u_{0}$ as a function of the increasing separation distance $D$. Different colors indicate different measurements on the same contact position. (b) Force $F / R$ normalized by the surface radius $R$ as a function of $D$. The black solid line is the model force $F(D) / R=2 \pi G_{0}(D)$ calculated for $K_{22}=10 \mathrm{pN}$ and $L / p \leq 0.1$ (Eqs 1 and 5). Circles indicate the position $D$ where a twist transition was observed by MBI. (c) Full-range force plot showing the adhesion force $F_{0}$ and outward jump from contact (arrow). The data correspond to those of Fig. 4.

\section{Conclusions}

We were able to measure the opto-mechanical response and reveal structural transitions of CLC films confined between solid surfaces using the SFA. The experimental data could be explained using a model of CLC deformation that included a finite strength of the surface anchoring, surface curvature and the presence of dislocation loops. Remarkably, the azimuthal strength of the planar anchoring on muscovite mica was found to be $W>10 \mathrm{~mJ} / \mathrm{m}^{2}$, which is orders of magnitude higher than 
typical values measured on polymers and other aligning surfaces. ${ }^{52}$ This finding are in line with recent SFA-MBI measurements indicating that thermotropic liquid crystals containing cyano-phenyls have a particularly strong azimuthal anchoring on mica. ${ }^{46}$

In our experiments, the surface were progressively separated and dislocation loops eventually annihilated at the contact position without showing any specific effect related to a non-zero line tension. The structural forces measured were weak but in agreement with the prediction of our model.

Surprisingly, these forces were orders of magnitude weaker than those measured during surface approach, i.e. there was a very large force hysteresis in a cycle of surface approach/retraction. Hysteresis originated from the process of nucleating (during surface approach) and annihilating (during retraction) a dislocation loop and was likely due to the presence of a large energy of defect nucleation leading to metastable states during surface approach. These observations will be the topic of an upcoming article.

\section{Conflicts of interest}

There are no conflicts to declare.

\section{Acknowledgements}

This work has been funded by the UK Royal Society under the International Exchanges Scheme, EU Framework Programme Horizon 2020 COST Action MP1303 ("Understanding and Controlling Nano and Mesoscale Friction"), and the European Research Council under Starting Grant no. 676861, LIQUISWITCH. We are grateful to Maria De Santo at the University of Calabria for kindly providing the liquid crystal materials. We also would like to thank Roberto Termine and Alfredo Pane at CNR-Nanotec for their assistance in thermal evaporation of silver on mica, and Marco Balabajew at the Unversity of Oxford for synchronizing the frames of the CCD camera.

\section{References}

1. P. G. De Gennes and J. Prost, The Physics of Liquid Crystals, Oxford University Press, Oxford, UK, 1993.

2. H. Kitzerow and C. Bahr, Chirality in Liquid Crystals, Springer, New York, USA, 2001.

3. M. F. Moreira, I. C. S. Carvalho, W. Cao, C. Bailey, B. Taheri and P. Palffy-Muhoray, Appl Phys Lett, 2004, 85, 2691 2693.

4. I. Sage, Liq Cryst, 2011, 38, 1551-1561.

5. L. Y. Zhao, Y. Wang, Y. G. Yuan, Y. J. Liu, S. Q. Liu, W. M. Sun, J. Yang and H. Y. Li, Opt Commun, 2017, 402, 181185.

6. R. A. M. Hikmet and H. Kemperman, Nature, 1998, 392, 476-479.

7. M. Mitov and N. Dessaud, Nat Mater, 2006, 5, 361-364.
8. N. Y. Ha, Y. Ohtsuka, S. M. Jeong, S. Nishimura, G. Suzaki, Y. Takanishi, K. Ishikawa and H. Takezoe, Nat Mater, 2008, 7, 43-47.

9. D. K. Yang and S. T. Wu, Fundamentals of liquid crystal devices, Wiley, Chichester, UK, 2014.

10. J. Xiang, Y. N. Li, Q. Li, D. A. Paterson, J. M. D. Storey, C. T. Imrie and O. D. Lavrentovich, Adv Mater, 2015, 27, 30143018.

11. H. Khandelwal, A. P. H. J. Schenning and M. G. Debije, Adv Energy Mater, 2017, 7.

12. H. Coles and S. Morris, Nat Photonics, 2010, 4, 676-685.

13. W. Feng, D. J. Broer and D. Q. Liu, Adv Mater, 2018, 30.

14. A. J. J. Kragt, D. J. Broer and A. P. H. J. Schenning, $A d v$ Funct Mater, 2018, 28.

15. J. E. Stumpel, E. R. Gil, A. B. Spoelstra, C. W. M. Bastiaansen, D. J. Broer and A. P. H. J. Schenning, Adv Funct Mater, 2015, 25, 3314-3320.

16. Y. H. Huang, Y. Zhou, C. Doyle and S. T. Wu, Opt Express, 2006, 14, 1236-1242.

17. H. G. Yoon, N. W. Roberts and H. F. Gleeson, Liq Cryst, 2006, 33, 503-510.

18. M. Y. Jeong and K. Kwak, Appl Optics, 2016, 55, 93789383.

19. H. Finkelmann, S. T. Kim, A. Munoz, P. Palffy-Muhoray and B. Taheri, Adv Mater, 2001, 13, 1069-+.

20. C. Bourgerette, B. Chen, H. Finkelmann, M. Mitov, J. Schmidtke and W. Stille, Macromolecules, 2006, 39, 81638170.

21. A. Varanytsia, H. Nagai, K. Urayama and P. PalffyMuhoray, Sci Rep, 2015, 5, 2-9.

22. V. A. Belyakov, I. W. Stewart and M. A. Osipov, Phys Rev E, 2005, 71.

23. V. A. Belyakov, S. V. Semenov and D. V. Shmeliova, Mol Cryst Liq Cryst, 2012, 559, 31-38.

24. S. Furumi, S. Yokoyama, A. Otomo and S. Mashiko, Appl Phys Lett, 2003, 82, 16-18.

25. I. P. Pinkevich, V. Y. Reshetnyak, Y. A. Reznikov and L. G. Grechko, Mol Cryst Liq Cryst, 1992, 223, 269-278.

26. D. Kasyanyuk, P. Pagliusi, A. Mazzulla, V. Reshetnyak, Y. Reznikov, C. Provenzano, M. Giocondo, M. Vasnetsov, O. Yaroshchuk and G. Cipparrone, Sci Rep, 2016, 6.

27. J. N. Israelachvili and P. M. McGuiggan, J Mater Res, 1990, 5, 2223-2231. .

28. R. Lhermerout and S. Perkin, Phys Rev Fluids, 2018, 3, 014201.

29. J. N. Israelachvili, R. G. Horn and E. Perez, J Physique (Paris), 1981, 42, 39.

30. S. H. J. Idziak, C. R. Safinya, R. S. Hill, K. E. Kraiser, M. Ruths, H. E. Warriner, S. Steinberg, K. S. Liang and J. N. Israelachvili, Science, 1994, 264, 1915-1918.

31. L. Moreau, P. Richetti and P. Barois, Phys Rev Lett, 1994, 73, 3556-3559.

32. P. Richetti, P. Kekicheff and P. Barois, J Physique II (France), 1995, 5, 1129-1154.

33. S. H. J. Idziak, I. Koltover, P. Davidson, M. Ruths, Y. L. Li, J. N. Israelachvili and C. R. Safinya, Physica B, 1996, 221, 289-295.

34. S. H. J. Idziak, I. Koltover, J. N. Israelachvili and C. R. Safinya, Phys Rev Lett, 1996, 76, 1477-1480.

35. I. Koltover, S. H. J. Idziak, P. Davidson, Y. Li, C. R. Safinya, M. Ruths, S. Steinberg and J. N. Israelachvili, I Physique II (France), 1996, 6, 893-907. 
36

P. Richetti, L. Moreau, P. Barois and P. Kekicheff, Phys Rev E, 1996, 54, 1749-1762.

37. M. Ruths, S. Steinberg and J. N. Israelachvili, Langmuir, 1996, 12, 6637-6650.

38.

J. Janik, R. Tadmor and J. Klein, Langmuir, 1997, 13, 44664473.

39. A. Artsyukhovich, L. D. Broekman and M. Salmeron, 73 Langmuir, 1999, 15, 2217-2223.

40. 8822-8829.

41. M. Ruths and S. Granick, Langmuir, 2000, 16, 8368-8376.

42. 5485

43. M. Ruths, M. Heuberger, V. Scheumann, J. J. Hu and W. Knoll, Langmuir, 2001, 17, 6213-6219.

44. B. Zappone, P. Richetti, R. Barberi, R. Bartolino and H. T. Nguyen, Phys Rev E, 2005, 71.

45. M. Ruths and B. Zappone, Langmuir, 2012, 28, 8371-8383.

46. B. Zappone, W. C. Zheng and S. Perkin, Rev Sci Instrum, 2018, 89.

47. M. Skarabot, Z. Lokar, K. Gabrijelcic, D. Wilkes and I. Musevic, Liq Cryst, 2011, 38, 1017-1020.

48. G. Barbero, W. Zheng and B. Zappone, J Mol Liq, 2017, 267, 242-248.

49. I. I. Smalyukh and O. D. Lavrentovich, Phys Rev E, 2002, 66, 051703.

50. I. I. Smalyukh and O. D. Lavrentovich, Phys Rev Lett, 2003, 90.

51. J. N. Israelachvili, Intermolecular and surface forces, Academic press, Waltham, MA, USA, 2011.

52. B. Jerome, Rep Prog Phys, 1991, 54, 391-451.

53. N. Katsonis, E. Lacaze and A. Ferrarini, J Mater Chem, 2012, 22, 25490-25490.

54. P. Sleczkowski, Y. Zhou, S. lamsaard, J. J. de Pablo, N. Katsonis and E. Lacaze, P Natl Acad Sci USA, 2018, 115, 4334-4339.

55. A. C. Callan-Jones, R. A. Pelcovits, R. B. Meyer and A. F. Bower, Phys Rev E, 2007, 75, 1-8.

56. K. Miyagi and Y. Teramoto, J Mater Chem C, 2018, 6 1370-1376.

57. I. I. Smalyukh, Annu Rev Cond Matt Phys, 2018, 9, $207-$ 226.

58. D. Marenduzzo, E. Orlandini and J. M. Yeomans, Phys Rev Lett, 2004, 92, 1-4.

59. D. Marenduzzo, E. Orlandini and J. M. Yeomans, J Chem Phys, 2006, 124, 204906.

60 F. Fadda, G. Gonnella, D. Marenduzzo, E. Orlandini and A Tiribocchi, J Chem Phys, 2017, 147, 064903.

61. I. Lelidis, G. Barbero and A. L. Alexe-lonescu, Phys Rev $E_{,}$ 2013, 87.

62. P. Pieranski and B. Jérôme, Phys Rev A, 1989, 40, 317-322.

63. I. Gryn, E. Lacaze, R. Bartolino and B. Zappone, Adv Funct Mater, 2015, 25, 142-149.

64. B. Jerome and Y. R. Shen, Phys Rev E, 1993, 48, 45564574.

65. B. Jerome, J. Obrien, Y. Ouchi, C. Stanners and Y. R. Shen Phys Rev Lett, 1993, 71, 758-761.

66. P. C. Schuddeboom and B. Jerome, Phys Rev E, 1997, 56, 4294-4305.

67. P. Rabinowitz, J Opt Soc Am A, 1995, 12, 1593-1601.

68. J. N. Israelachvili, J Coll Interf Sci, 1973, 44, 259-272.

69. R. G. Horn and D. T. Smith, Appl Optics, 1991, 30, 59
70.

K. M. McPeak, S. V. Jayanti, S. J. P. Kress, S. Meyer, S. lotti, A. Rossinelli and D. J. Norris, ACS Photonics, 2015, 2, 326333.

71. A. D. Kiselev and T. J. Sluckin, Phys Rev E, 2005, 71.

72. L. Blinov and V. Chigrinov, Electrooptic Effects in Liquid Crystal Materials, Springer-Verlag, New York, 1994.

B. Klus, U. A. Laudyn, M. A. Karpierz and B. Sahraoui, Opt Express, 2014, 22, 30257-30266.

R. P. Sun, X. M. Huang, K. Ma, Z. K. Wang and J. Min, Phys Rev $E, 1994$, 50, 1253-1256.

Y. Cui, Y. C. Yang and D. K. Yang, Jpn J Appl Phys, 2015, 54. 